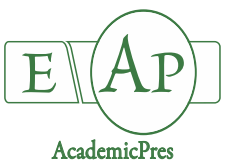

Ayaz Tilkat E et al. (2020)
Notulae Botanicae Horti Agrobotanici Cluj-Napoca 48(4):1885-1900
DOI:10.15835/48412016
Research Article

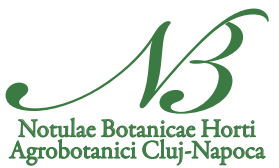

\title{
Antioxidant and antimicrobial responses associated with in vitro salt stress of in vitro and in vivo grown Pistacia khinjuk stocks
}

\author{
Emine AYAZ TİLKAT ${ }^{1 *}$, Nesrin HAŞİMİ ${ }^{1}$, İbrahim S. KURU ${ }^{1}$, \\ Veysel SÜZERER ${ }^{2,3}$
}

\author{
${ }^{1}$ Batman University, Science \& Literature Faculty, Department of Biology, Batman, \\ Turkey; eayaztilkat@gmail.com ("correspondingauthor);nesrin.hasimi@batman.edu.tr \\ ${ }^{2}$ Batman University, Sason Vocational School, Department of Crop and Animal Production, Batman, \\ Turkey; ibrahimselcuk.kuru@batman.edu.tr \\ ${ }^{3}$ Bingöl University, Vocational School of Health Services, Department of Pharmacy Services-Bingöl, Turkey; beyso1985@gmail.com
}

\begin{abstract}
P. khinjuk Stocks, known as Bittım or Buttum in Turkey, is a member of the Anacardiaceae family. The essential oil of khinjuk pistachio has been used to treat various illnesses because of their anti-inflammatory, anticancer, antipyretic, antibacterial, anthelmintic, antiviral effects in various folk medicines. At the same time, fruits of khinjuk pistachio are used as edible wild fruits. In this study, it was aimed to determine and compare the antibacterial, antioxidant activities and total phenolic and flavonoid amounts of different parts (root, stem and leaf explants) of in vivo (grown naturally) and in vitro derived khinjuk pistachio plants under salt $(\mathrm{NaCl})$ stress. Ethanol extracted explants were used for performing biological and chemical parameters. According to the results, generally, in vivo samples shows higher antioxidant and antimicrobial activity besides the higher number of phenolic compounds than their counterparts in vitro. We have also determined that the biological activity of in vitro salt elicited explants was higher than in vitro control explants. Generally, both female and male in vivo samples have higher antioxidants (DPPH, ABTS, CUPRAC) and antimicrobial activities than in vitro samples. The various plant parts (root, stem, leaf) belonging to both in vivo and in vitro samples have different biological activity level. In terms of antimicrobial activity, female plant extracts are more active than all other tested extracts. As a result, although increased salinity values significantly reduced antimicrobial activity, it is determined that $100 \mathrm{mM} \mathrm{NaCl}$ applications to in vitro leaf extracts exhibited moderate antimicrobial activity against $S$. aureus and $C$. albicans.
\end{abstract}

Keywords: antimicrobial; antioxidant; P. khinjuk Stocks; salt stress

\section{Introduction}

Pistacia, a genus of flowering plants from the family Anacardiaceae, contains about twenty species; among them, five are more well-known, including $P$. vera, $P$. atlantica, $P$. terebinthus, $P$. khinjuk, and $P$. lentiscus. The species of the genus Pistacia are evergreen or deciduous resin-bearing shrubs and trees, which are characterized as xerophytic trees and growing to $8-10 \mathrm{~m}$ tall. The leaves are alternate, pinnately compounds, and can be either evergreen or deciduous, depending on species. The genus Pistacia has been used by many 
civilizations from prehistoric times to the present day for various purposes due to their resin, fruit, leaves, and chemical components. The potential of antioxidant, anthelmintic, antimicrobial, anti-inflammatory, and cytotoxic effects of Pistacia species, primarily due to flavonoids and other secondary metabolites, have always attracted the attention of researchers. So far, there are many studies on the antimicrobial, antioxidant, antiinflammatory, cytotoxicity antiangiogenic, etc. properties of this genus in the literature.

For example, under the light of literature, a flavone showed high antioxidant activity was identified as well as apigenin, luteolin, and other flavonoids from the extracts of $P$. terebinthus fruits (Topçu, 2007). In another study, antimicrobial and antioxidant characteristics of the extracts of the resin of $P$. lentiscus has also been reported (Giaginis and Theocharis, 2011). Also, Gallotannin, a kind of tannin, has more antioxidant potential isolated from the leaf extract of $P$. weinmannifolia, called "Pistafolia A" (Wei et al., 2002). Furthermore, it was shown that the ethanol extracts of fruits and leaves of $P$. vera L. have a higher antioxidant effect than the resin, according to a study by Hosseinzadeh et al. (2012). It was also determined by the MIC test that $P$. terebinthus $\mathrm{L}$. volatile components significantly inhibited the growth of different organisms such as Shigella dysenteriae, Escherichia coli, Bacillus subtilis and Pseudomonas aeruginosa (Holley and Patel, 2005; Mohagheghzadeh et al., 2010). These studies mentioned above show that Pistacia species can use as an essential natural antioxidant and antimicrobial source. At the same time, it was reported that not only the seeds but also other parts of naturally grown $P$. khinjuk Stocks could be a source of phenolics and flavonoids compounds (Hacibekiroglu et al., 2015, Hatamnia et al., 2016; Ahmed et al., 2017; Hazrati et al., 2020). However, many of chemical and biological activity studies were found in the literature on extracted explants for other economically important plant species (Esmat et al., 2012; Mirian et al., 2014; Tahvilian et al., 2016; Ahmed et al., 2017; Taghizadeh et al., 2018) there is no study about chemical and biological activity on in vitro grown plants under salt stress conditions. Although many studies confirm the negative effect of salinity on growth, it could be led to an increase in the production of secondary plant metabolites, antioxidant and antimicrobial effects.

Moreover, the enhanced synthesis of these secondary metabolites under salt stress conditions is believed to protect the cellular structures from oxidative effects. To avoid oxidative damage resulting from salt stress, higher plants have developed different adaptive mechanisms through the biosynthesis of a cascade of antioxidants. Indeed, polyphenolic compounds participate in the defence against reactive oxygen species (ROS), which are inevitably produced when environmental stresses impair aerobic or photosynthetic metabolism. It has been proven that the amount of antioxidant and antimicrobial components increases in adverse environmental conditions in plant tissues (Salem et al., 2013).

In the light of the literature review about the effect of salt stress on Pistacia species, it has been found that the articles are mostly related to physiological studies, and generally, medium and high salt applications negatively affect growth and increase soluble sugar and proline content (Rahneshan et al., 2018).

Considering the studies investigating the physiological responses of in vitro salt stress on the Pistacia genus, in a study on two different species ( $P$. vera L. and $P$. atlantica Desf.), the seeds were cultured for 25 days at different $\mathrm{NaCl}$ concentrations $(0,40,60,80,131,158.5$ and $240 \mathrm{mM})$. It was noted that low $\mathrm{NaCl}$ applications did not cause plant death in any sample, but under high salt conditions (158.5 and $240 \mathrm{mM}$ ), 20$25 \%$ mortality was observed. Besides, regarding salinity effects 60 and $80 \mathrm{mM} \mathrm{NaCl}$ levels caused significant reductions in stem growth and leaf number in $P$. vera species. However, salinity between 40 and $80 \mathrm{mM} \mathrm{NaCl}$ caused a reduction in the number of roots of both species. After 45 days of culture, fresh weights also decreased significantly, and it was observed that high $\mathrm{NaCl}$ applications $(131-240 \mathrm{mM} \mathrm{NaCl})$ caused a significant increase in proline content in both Pistacia species (Chelli-Chaabouni et al., 2010).

In another study, the salinity tolerance of pistachio (Pistacia vera L.), embryos were investigated. The embryos developed from mature seeds were isolated and cultured in vitro and subjected to different $\mathrm{NaCl}$ concentrations $(0,42.8,85.5,171.1$ and $256.6 \mathrm{mM})$ for 30 days. According to the results in vitro germination of embryonic axes was not affected by the salt concentration. However, the germinated embryo survival rates 
decreased from 100\% for the control to 62.9\% for the highest salt concentration (256.6 mM) (Benmahioul et al., 2009).

Another study (Ayaz Tilkat et al., 2019) reported that the effect of different sodium chloride $(\mathrm{NaCl})$ concentrations $(0,50,100,150,200,250 \mathrm{mM})$ on growth and physiological parameters of Pistacia lentiscus L. seedlings raised in in vitro condition for four weeks was investigated. According to the study, the morphological, physiological and biochemical changes that occurred in the seedlings were measured and recorded after exposure to salt stress. The results indicate that the visible leaf damage of Lentisk seedlings is affected by high salt concentrations. High salinity concentrations significantly reduce root and stem lengths, relative water content (RWC), total chlorophyll, $\mathrm{Chl} a, \mathrm{Chl} b$ and carotenoid values after the culture periods. At $250 \mathrm{mM}$ salt concentration, root and stem growth were found to be stopped entirely. Consequently, the parameters that over the $50 \mathrm{mM}$ salt concentrations are caused in a decrease in the activity of the antioxidant enzyme peroxidase (POD).

There is only one study in the literature investigating the physiological effects of salt stress applied in vitro in khinjuk pistachio (Ayaz Tilkat et al., 2017). In this study, mature khinjuk pistachio seeds cultured in in-vitro conditions were exposed to different salt $(\mathrm{NaCl})$ parameters $(0,50,100,150,200,250 \mathrm{mM})$. The seeds germinated in PGR-free MS (Murashige and Skoog, 1962) medium containing different concentrations of $\mathrm{NaCl}$ were exposed to salt stress for four weeks. As a result, it was determined that there is a positive correlation between soluble carbohydrate values and POD activities due to the increased $\mathrm{NaCl}$ concentration (Ayaz Tilkat et al., 2017).

All these studies aside, in this context, the current study aims to investigate and compare the antioxidant and antimicrobial properties of the root, stem, and leaf extracts obtained from the plantlets germinated under different salt concentrations in vitro, and the same parts extract from in vivo grown plants. Antimicrobial activity screening was determined using the disc diffusion method, and minimal inhibitory concentration (MIC) values were defined. The antioxidant activity potential of the extracts was evaluated by different methods, namely, Folin-Ciocalteu (FCR), 1,1-diphenyl-2-picrylhydrazyl (DPPH) 2,2'-azinobis-(3ethylbenzothiazoline-6-sulfonic acid) (ABTS) radical scavenging capacity tests and cupric ion reducing antioxidant capacity (CUPRAC) method.

Since there is no study in the literature about antimicrobial and antioxidant activities of in vivo and in vitro samples of khinjuk pistachio, the topic is highly original, and the results will be reported for the first time.

\section{Materials and Methods}

\section{Biological material}

In this study, leaves, roots, and stems of mature male and female $P$. khinjuk Stocks trees raised in Gaziantep Pistachio Research Institute were used for obtaining in vivo extracts, and mature seeds of this trees were used as in-vitro salt stress experiments for antioxidant and antimicrobial activity studies. The protocols developed by Tilkat et al. (2005) were modified for in vitro micropropagation of seeds. The diagram of all the processes in the study was shown in Figure 1.

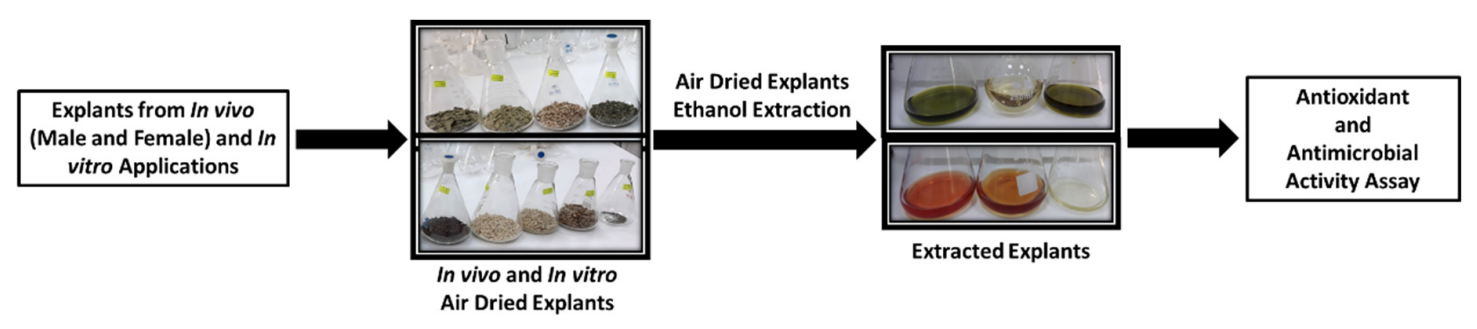

Figure 1. Diagram of the plant extraction process 


\section{Harvesting in-vivo explants}

The root, stem, and leaves originated mature female, and male khinjuk pistachio was harvested from the orchards of Gaziantep Pistachio Research Institute in July 2019, then dried at room temperature in the dark, pounded using pestles, and wooden mortars separately for using in the extraction procedures.

\section{Obtaining of in-vitro explants under salt stress}

For all the stages of surface sterilization of seeds, culture initiation, and proliferation of seedlings, the protocol developed by Tilkat et al. (2005) was modified and used. The seeds were surface sterilized by immersion in a $20 \%(\mathrm{w} / \mathrm{v})$ commercial bleach solution $(\mathrm{NaOCl})$ for $20 \mathrm{~min}$. In this context, the seed coats were then removed, and the kernels were washed three times with sterile distilled water before inoculating onto the MS basal medium. The plant growth regulator (PGR)-free MS basal medium was supplemented with 100 $\mathrm{mg} / \mathrm{l}$-ascorbic acid, $3 \%$ sucrose $(\mathrm{w} / \mathrm{v})$, and solidified with agar $(0.7 \%, \mathrm{w} / \mathrm{v})$. The media were adjusted to $\mathrm{pH}$ 5.7 by using hydrochloric acid $(\mathrm{HCl})$ at $0.1 \mathrm{~N}$ and sodium hydroxide $(\mathrm{NaOH})$ at $0.5 \mathrm{~N}$ before autoclaving $(120$ ${ }^{\circ} \mathrm{C}$ for $\left.20 \mathrm{~min}\right)$. Cultures were maintained at $25 \pm 2{ }^{\circ} \mathrm{C}$ with a $16 \mathrm{~h}$ photoperiod $\left(40 \mu \mathrm{mol} \mathrm{m} \mathrm{s}^{-1}\right)$. Almost $1 \mathrm{~cm}$ long shoots of in vitro cultivated $P$. khinjuk Stocks, were taken from axenic stock cultures and transferred into MS medium containing different $\mathrm{NaCl}$ concentrations $(0,50,100,200 \mathrm{mM})$ with a control group for a culture period (28 days). Leaf, root, and stem parts of seedlings obtained in vitro were isolated separately after four weeks to use in the extraction procedures.

\section{Plant extraction}

In vivo and in vitro, plant samples were dried in the dark at room temperature and powdered. The material was macerated with ethanol a few times, and then the solvent was evaporated, and the crude extract was obtained.

\section{Antioxidant activity assays}

The antioxidant activity of the extracts was determined by three different methods, including DPPH free radical (Blois, 1958; Ongphimai, 2013), ABTS cation radical scavenging activity (Re et al., 1999) and cupric reducing antioxidant capacity (CUPRAC) (Apak et al., 2004). Ascorbic Acid (AA), Butylated Hydroxyanisole (BHA) and Butylated Hydroxytoluen (BHT) and were used as positive controls.

\section{DPPH method}

In DPPH method different concentrations $(10,25,50,100 \mu \mathrm{g} / \mathrm{ml})$ of extracts prepared. $4 \mathrm{ml}$ of $0.004 \%$ $\mathrm{DPPH} /$ ethanol solution and $1 \mathrm{ml}$ of extract was mixed in the test tube and were kept at room temperature for $30 \mathrm{~min}$. After the incubation period, the absorbance (A) was measured at $517 \mathrm{~nm}$. The inhibition \% (I\%) was calculated by the following equation:

Inhibition $\%=\left[\mathrm{A}_{\text {control }}-\mathrm{A}_{\text {sample }} / \mathrm{A}_{\text {control }}\right] \times 100$

\section{ABTS method}

In ABTS method, different concentrations $(10,25,50,100 \mu \mathrm{g} / \mathrm{ml}$ ) of extracts prepared. $7 \mathrm{mM}$ ABTS solution was adjusted to 0.7 absorbances at $734 \mathrm{~nm} .4 \mathrm{ml}$ of ABTS solution and $1 \mathrm{ml}$ of the extract have mixed in the test tube and were kept at room temperature for $30 \mathrm{~min}$. After the incubation period, the absorbance (A) was measured at $734 \mathrm{~nm}$. The inhibition (I\%) was calculated by the following equation:

Inhibition $\%=\left[\mathrm{A}_{\text {control }}-\mathrm{A}_{\text {sample }} / \mathrm{A}_{\text {control }}\right] \times 100$ 


\section{CUPRAC method}

In CUPRAC method, different concentrations $(10,25,50,100 \mu \mathrm{g} / \mathrm{ml})$ of extracts prepared. $1 \mathrm{ml}$ of $\mathrm{CuCl}_{2}$ solution, $1 \mathrm{ml}$ of neocuproine solution and $1 \mathrm{ml}$ of $\mathrm{NH}_{4} \mathrm{CH}_{3} \mathrm{CO}_{2}$ solution were mixed in the test tube. $1 \mathrm{ml}$ of extract was added to tube content. After the 60 minutes of the incubation period, the absorbance was measured at $450 \mathrm{~nm}$. The absorbance values of the samples were evaluated against the control. The increased absorbance value indicates increased activity.

\section{The total phenolic and flavonoid content}

The total phenolic and flavonoid content of the extracts was determined using the Folin-Ciocalteu reagent (FCR) (Slinkard and Singleton, 1977) and by the aluminium nitrate method (Moreno et al., 2000), respectively. Total phenolic and flavonoid contents in the crude extracts expressing as Gallic acid and quercetin equivalents.

$4.6 \mathrm{ml}$ of different concentrations of gallic acid was incubated with $0.1 \mathrm{ml} \mathrm{FCR}$ for $3 \mathrm{~min} .0 .3 \mathrm{ml}$ of $2 \%$ $\mathrm{Na}_{2} \mathrm{CO}_{3}$ solution was added to a test tube and incubated for 2 hours. After then the absorbance was measured at $760 \mathrm{~nm}$. A standard curve was plotted, and the amount of total phenolic content was calculated according to the following equations:

Absorbance $=0.0356$ gallic acid $(\mu \mathrm{g})-0.0047(\mathrm{R} 2=0.9970)$

$4.8 \mathrm{ml}$ of different concentrations of quercetin was incubated with $0.1 \mathrm{ml}$ of $1 \mathrm{M} \mathrm{CH}_{3} \mathrm{CO}_{2} \mathrm{~K}$ for $60 \mathrm{~min}$. $0.1 \mathrm{ml}$ of $10 \% \mathrm{Al}\left(\mathrm{NO}_{3}\right)_{3}$ solution was added to the test tube and incubated for $40 \mathrm{~min}$. After then the absorbance was measured at $415 \mathrm{~nm}$. A standard curve was plotted, and the amount of total phenolic content was calculated according to the following equations:

Absorbance $=0.0619$ quercetin $(\mu \mathrm{g})-0.0043(\mathrm{R} 2=0.9991)$

\section{Antimicrobial activity}

The antimicrobial activity of the extracts was evaluated by the disc diffusion method (NCCLS, 1997) and minimum inhibitory concentrations (MIC) (NCCLS, 2009). Five microorganisms (Escherichia coli ATCC25922, Pseudomonas aeroginosa ATCC27853, Staphylococcus aureus ATCC25923, Streptococcus pyogenes ATCC19615 and Candida albicans ATCC10231) were used for antimicrobial assays. Ampicillin and nystatin were used as positive controls. The nutrient broth medium was inoculated with each microorganism and incubated for $12-16$ hours at $37^{\circ} \mathrm{C} .100 \mu \mathrm{l}$ of the test microorganisms prepared overnight culture was spread into plates containing nutrient agar. Then $15 \mu \mathrm{l} \mathrm{of} 100 \mathrm{mg} / \mathrm{ml}$ concentrations of all extracts were prepared and impregnated on sterile paper discs placed in plates. Inhibition zone diameters were measured after 24 hours' incubation at $37^{\circ} \mathrm{C}$ for bacteria and 48 hours' incubation at $30^{\circ} \mathrm{C}$ for yeast. The active extracts were then processed to determine the MIC value. $100 \mu \mathrm{l}$ serial dilutions of extracts, $90 \mu \mathrm{l}$ broth, and $10 \mu \mathrm{l}$ microorganism overnight cultures (turbidity equal to $0.5 \mathrm{McFarland}$ ) were pipetted into 96 well sterile plates. After 24 hours incubation at the appropriate temperature, the wells were evaluated. $100 \mu \mathrm{l}$ was taken from the lowest concentration well without visible growth and spread on solid medium. After incubation at the appropriate temperature and time mentioned above, MIC values were determined according to the number of colonies on the plate.

\section{Statistical analysis}

All the experiments were conducted using a completely randomized block design, and they were performed in triplicate. Statistical analysis was performed using SPSS 25.0 Statistical program. One-way ANOVA was used for the analysis and mean values were compared by employing Tukey's test at $\mathrm{p} \leq 0.05$ probability level. 


\section{Results}

The modified in vitro micropropagation protocol developed by Tilkat et al. (2005) was applied to $P$. khinjuk Stocks seeds to initiate shoot cultures, and successful results were obtained (Figure 2).

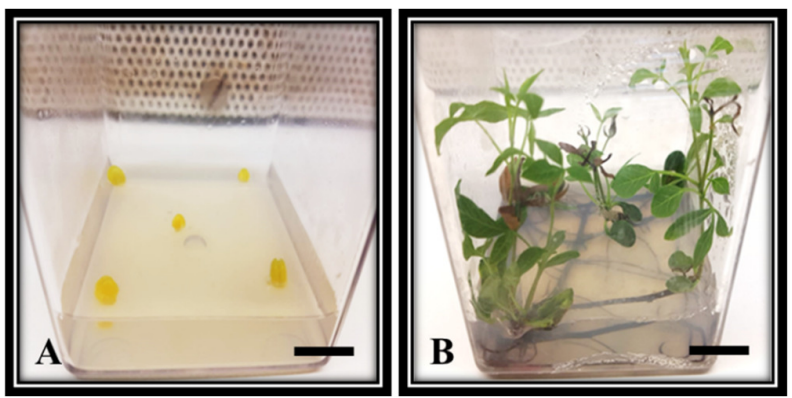

Figure 2. Propagation of $P$. khinjuk Stocks from seeds

A) Mature seeds inoculated on PGR-free MS culture medium, B) The seedlings, four weeks after culturing in vitro (Bars: $0.9 \mathrm{~cm}$ )

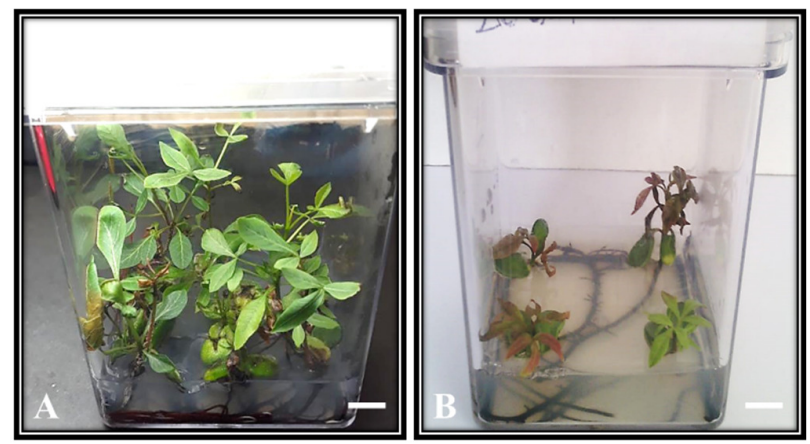

Figure 3. The seedlings germinated in vitro under different $\mathrm{NaCl}$ applications A) $50 \mathrm{mM} \mathrm{NaCl}$ application, B) $200 \mathrm{mM} \mathrm{NaCl}$ application

In general, it was seen that healthy shoot growth was obtained from seeds belonging to the control group, whereas seeds elicited by different salt concentrations show weaker shoot growth. The observations indicate that the shoot growth of seeds was reduced by salt application $(50,100$, and $200 \mathrm{mM})$. Parallel to increasing salt concentrations, stem number, and root-stem-leaf dry weights were decreased; especially chlorosis was detected in shoots. Antioxidant and antimicrobial activity result of in vitro seedlings, which are elicited in 3 different salt concentrations and in vivo leaves, roots, and stems of male and female $P$. khinjuk Stocks were separately investigated and presented in tables below.

We observed a strong positive correlation between TPC in the plant and its antioxidant activity, which suggests that phenolic compounds significantly contribute to this antioxidant activity.

Assessment of DPPH activity, generally in vivo male and female samples were showed higher activity than in vitro elicited seedling explants. Applied minimum salt concentration to in vitro male root was showed higher activity $(56.80 \pm 1.50 / 10 \mu \mathrm{g})$ compare from other genotype samples. Between in vitro elicited seedling samples was observed highest activity in $50 \mathrm{mM} \mathrm{NaCl}$ leaf sample $(39.67 \pm 0.51 / 10 \mu \mathrm{g})$ (Table 1). When evaluated in terms of antioxidant activity in the root part, there is a significant increase compared to the control together with salt concentration increases. 
Table 1. Results of antioxidant activity by DPPH method (I \%)

\begin{tabular}{|c|c|c|c|c|c|c|}
\hline \multicolumn{3}{|c|}{ Explant Type } & $10 \mu \mathrm{g} / \mathrm{ml}$ & $25 \mu \mathrm{g} / \mathrm{ml}$ & $50 \mu \mathrm{g} / \mathrm{ml}$ & $100 \mu \mathrm{g} / \mathrm{ml}$ \\
\hline \multirow{6}{*}{$\begin{array}{l}5 \\
0 \\
0 \\
\simeq\end{array}$} & \multirow{2}{*}{ 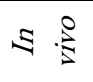 } & Male & $56.80 \pm 1.50 \mathrm{bC}$ & $78.30 \pm 1.67 \mathrm{~b} \mathrm{~B}$ & $91.05 \pm 1.31 \mathrm{bA}$ & $92.29 \pm 0.50 \mathrm{c} \mathrm{A}$ \\
\hline & & Female & $48.81 \pm 1.14 \mathrm{cD}$ & $78.30 \pm 1.29 b \mathrm{~b}$ & $90.29 \pm 0.90 \mathrm{~b} \mathrm{~B}$ & $92.29 \pm 0.48 \mathrm{c} \mathrm{A}$ \\
\hline & \multirow{4}{*}{$\Sigma: \stackrel{:}{:}$} & Control & $17.72 \pm 0.87 \mathrm{~d} D$ & $21.68 \pm 0.73$ е C & $23.09 \pm 0.51 \mathrm{e} \mathrm{B}$ & $25.15 \pm 0.29 \mathrm{fA}$ \\
\hline & & $50 \mathrm{NaCl}$ & $22.93 \pm 1.02 \mathrm{~dB}$ & $24.64 \pm 0.18 \mathrm{de} A$ & $25.02 \pm 0.65$ e $A$ & $26.35 \pm 0.68 \mathrm{fA}$ \\
\hline & & $100 \mathrm{NaCl}$ & $22.83 \pm 0.94 \mathrm{~d} D$ & $32.06 \pm 1.07 \mathrm{c} \mathrm{C}$ & $43.76 \pm 1.05 \mathrm{~dB}$ & $50.33 \pm 0.99$ е A \\
\hline & & $200 \mathrm{NaCl}$ & $19.98 \pm 0.53 \mathrm{~d} D$ & $28.54 \pm 0.62 \mathrm{~cd} \mathrm{C}$ & $54.32 \pm 0.70 \mathrm{cB}$ & $60.89 \pm 0.95 \mathrm{~d} \mathrm{~A}$ \\
\hline \multicolumn{3}{|c|}{ BHT } & $2.40 \pm 0.12$ e D & $4.90 \pm 0.19 \mathrm{fC}$ & $12.91 \pm 0.18 \mathrm{fB}$ & $26.42 \pm 0.25 \mathrm{fA}$ \\
\hline \multicolumn{3}{|c|}{ BHA } & $70.28 \pm 2.18$ a C & $82.64 \pm 1.57 \mathrm{~b} \mathrm{~B}$ & $94.35 \pm 1.24 \mathrm{~b} \mathrm{~A}$ & $95.40 \pm 0.42 \mathrm{~b} \mathrm{~A}$ \\
\hline \multicolumn{3}{|c|}{ AA } & $68.19 \pm 1.69$ a C & $96.13 \pm 0.68$ a B & $99.78 \pm 0.52 \mathrm{a} \mathrm{A}$ & $99.82 \pm 0.24 \mathrm{a} \mathrm{A}$ \\
\hline \multirow{6}{*}{$\underset{⿱ ㇒}{\sum_{\text {出 }}}$} & \multirow{2}{*}{$\Sigma . \stackrel{0}{5}$} & Male & $35.01 \pm 1.30 \mathrm{bD}$ & $54.42 \pm 1.00 \mathrm{dC}$ & $75.73 \pm 0.63 \mathrm{~dB}$ & $92.10 \pm 0.76 \mathrm{~cd} \mathrm{~A}$ \\
\hline & & Female & $30.06 \pm 1.04 \mathrm{bcD}$ & $43.86 \pm 0.59$ е C & $74.21 \pm 1.07 \mathrm{~dB}$ & $91.24 \pm 0.64 \mathrm{dA}$ \\
\hline & \multirow{4}{*}{$\Xi: \stackrel{:}{\Sigma}$} & Control & $21.02 \pm 0.65 \mathrm{~d} \mathrm{D}$ & $45.67 \pm 1.29$ е C & $74.02 \pm 0.77 \mathrm{~dB}$ & $88.01 \pm 0.26 \mathrm{eA}$ \\
\hline & & $50 \mathrm{NaCl}$ & $27.13 \pm 0.88 \mathrm{~cd} \mathrm{D}$ & $63.28 \pm 0.92 \mathrm{c} \mathrm{C}$ & $89.12 \pm 1.13 \mathrm{c} \mathrm{B}$ & $94.25 \pm 0.58 \mathrm{bc} \mathrm{A}$ \\
\hline & & $100 \mathrm{NaCl}$ & $25.68 \pm 1.11 \mathrm{~cd} \mathrm{D}$ & $37.96 \pm 0.66 \mathrm{fC}$ & $41.86 \pm 0.85$ e B & $66.79 \pm 0.40 \mathrm{fA}$ \\
\hline & & $200 \mathrm{NaCl}$ & $23.88 \pm 1.08 \mathrm{~d} D$ & $31.01 \pm 0.87 \mathrm{~g} \mathrm{C}$ & $35.01 \pm 0.49 \mathrm{fB}$ & $52.80 \pm 0.28 \mathrm{~g} \mathrm{~A}$ \\
\hline \multicolumn{3}{|c|}{ BHT } & $2.40 \pm 0.12$ e D & $4.90 \pm 0.19 \mathrm{~h} \mathrm{C}$ & $12.91 \pm 0.18 \mathrm{gB}$ & $26.42 \pm 025 \mathrm{~h} \mathrm{~A}$ \\
\hline \multicolumn{3}{|c|}{ BHA } & $70.28 \pm 2.18$ a C & $82.64 \pm 1.57 \mathrm{~b} \mathrm{~B}$ & $94.35 \pm 1.24 \mathrm{~b} \mathrm{~A}$ & $95.40 \pm 0.42 \mathrm{~b} \mathrm{~A}$ \\
\hline \multicolumn{3}{|c|}{$\mathrm{AA}$} & $68.19 \pm 1.69$ a C & $96.13 \pm 0.68 \mathrm{a} \mathrm{B}$ & $99.78 \pm 0.05$ a A & $99.82 \pm 0.04 \mathrm{a} \mathrm{A}$ \\
\hline \multirow{6}{*}{$\begin{array}{l}\text { 宏 } \\
\text { 孚 }\end{array}$} & \multirow{2}{*}{$\Xi \cdot \stackrel{0}{5}$} & Male & $39.20 \pm 1.49 \mathrm{~b} \mathrm{C}$ & $62.03 \pm 1.78 \mathrm{dC}$ & $86.96 \pm 1.06 \mathrm{c} \mathrm{B}$ & $92.38 \pm 0.56 \mathrm{c} \mathrm{A}$ \\
\hline & & Female & $45.57 \pm 1.76 \mathrm{~b} \mathrm{D}$ & $76.21 \pm 1.66 \mathrm{c} \mathrm{C}$ & $85.63 \pm 0.85 \mathrm{c} \mathrm{B}$ & $93.05 \pm 0.40 \mathrm{c} \mathrm{A}$ \\
\hline & \multirow{4}{*}{$\Sigma: \stackrel{:}{S}$} & Control & $32.82 \pm 0.50 \mathrm{bc} D$ & $44.33 \pm 1.06 \mathrm{fC}$ & $77.09 \pm 0.87 \mathrm{~dB}$ & $87.91 \pm 0.81 \mathrm{~d} A$ \\
\hline & & $50 \mathrm{NaCl}$ & $39.67 \pm 0.51 \mathrm{~b} \mathrm{D}$ & $53.66 \pm 0.98$ е C & $88.27 \pm 1.06 \mathrm{c} \mathrm{B}$ & $96.25 \pm 0.62 \mathrm{~b} \mathrm{~A}$ \\
\hline & & $100 \mathrm{NaCl}$ & $32.15 \pm 0.96 \mathrm{dD}$ & $38.15 \pm 0.38 \mathrm{gC}$ & $63.14 \pm 0.70$ е B & $86.48 \pm 0.48 \mathrm{~d} \mathrm{~A}$ \\
\hline & & $200 \mathrm{NaCl}$ & $29.40 \pm 1.11 \mathrm{dD}$ & $36.72 \pm 0.58 \mathrm{gC}$ & $43.57 \pm 0.39 \mathrm{fB}$ & $68.50 \pm 1.22 \mathrm{e} \mathrm{A}$ \\
\hline \multicolumn{3}{|c|}{ BHT } & $2.40 \pm 0.12$ e D & $4.90 \pm 0.19 \mathrm{~h} \mathrm{C}$ & $12.91 \pm 0.18 \mathrm{~g} \mathrm{~B}$ & $26.42 \pm 0.25 \mathrm{fA}$ \\
\hline \multicolumn{3}{|c|}{$\mathrm{BHA}$} & $70.28 \pm 2.18$ a C & $82.64 \pm 1.57 \mathrm{~b} \mathrm{~B}$ & $94.35 \pm 1.24 \mathrm{~b} \mathrm{~A}$ & $95.40 \pm 0.42 \mathrm{bc} \mathrm{A}$ \\
\hline \multicolumn{3}{|c|}{ AA } & $68.19 \pm 1.69$ a C & $96.13 \pm 0.68 \mathrm{a} \mathrm{B}$ & $99.78 \pm 0.51 \mathrm{aA}$ & $99.82 \pm 0.04 \mathrm{a} \mathrm{A}$ \\
\hline
\end{tabular}

Each value is the means \pm SE of 3 replicates measurement.

Means followed by different lower-case letters in the columns and upper-case letters in the rows showed significant differences, by the Tukey's test, at $\mathrm{p} \leq 0.05$

In vivo samples showed very high activity in the ABTS test system, which is far above both in vivo samples and positive controls. In both female and male plants (Table 2.), in vivo derived stem extracts showed relatively low activity compared to leaves and roots. Most of the in vitro samples were exhibited activity close to positive controls. Besides this, in vitro root extract has a deficient activity compared to stem and leaf extracts (Table 2.) In terms of in vitro plants compared to control, the highest values stand out as $99.26 \pm 0.13$ (control $55.88 \pm 1.39$ ) at $100 \mu \mathrm{g} / \mathrm{ml} 200 \mathrm{mM} \mathrm{NaCl}$ in root; $87.26 \pm 1.07$ (control $71.77 \pm 0.65)$ at $10 \mu \mathrm{g} / \mathrm{ml} 50 \mathrm{mM}$ $\mathrm{NaCl}$ in stem and $98.70 \pm 0.07$ (control $69.00 \pm 0.73$ ) at $10 \mu \mathrm{g} / \mathrm{ml} 50 \mathrm{mM} \mathrm{NaCl}$ in leaf. 
Ayaz Tilkat E et al. (2020). Not Bot Horti Agrobo 48(4):1885-1900

Table 2. Results of antioxidant activity by ABTS method (I\%)

\begin{tabular}{|c|c|c|c|c|c|c|}
\hline \multicolumn{3}{|c|}{ Explant type } & $10 \mu \mathrm{g} / \mathrm{ml}$ & $25 \mu \mathrm{g} / \mathrm{ml}$ & $50 \mu \mathrm{g} / \mathrm{ml}$ & $100 \mu \mathrm{g} / \mathrm{ml}$ \\
\hline \multirow{6}{*}{$\begin{array}{l}5 \\
0 \\
0 \\
\simeq 1\end{array}$} & \multirow{2}{*}{$\Xi$} & Male & $99.26 \pm 0.05$ a A & $99.26 \pm 0.14 \mathrm{aA}$ & - & - \\
\hline & & Female & $99.44 \pm 0.09 \mathrm{a} \mathrm{A}$ & $99.26 \pm 0.06 \mathrm{aA}$ & - & - \\
\hline & \multirow{4}{*}{$\begin{array}{l}D \\
: \\
\vdots \\
\vdots\end{array}$} & Control & $8.76 \pm 0.23 \mathrm{fg} D$ & $13.25 \pm 0.27 \mathrm{fC}$ & $20.74 \pm 0.47 \mathrm{fB}$ & $55.88 \pm 1.39$ е A \\
\hline & & $50 \mathrm{NaCl}$ & $10.33 \pm 0.46 \mathrm{fD}$ & $20.84 \pm 0.81 \mathrm{eC}$ & $29.33 \pm 0.55$ e B & $65.12 \pm 0.98 \mathrm{~d} \mathrm{~A}$ \\
\hline & & $100 \mathrm{NaCl}$ & $28.22 \pm 0.61 \mathrm{e} \mathrm{D}$ & $64.02 \pm 0.71 \mathrm{~d} \mathrm{C}$ & $92.61 \pm 0.47 \mathrm{~b} \mathrm{~B}$ & $96.67 \pm 0.44 \mathrm{bc} \mathrm{A}$ \\
\hline & & $200 \mathrm{NaCl}$ & $33.94 \pm 0.70 \mathrm{dD}$ & $66.42 \pm 0.42 \mathrm{dC}$ & $98.33 \pm 0.36 \mathrm{a} \mathrm{B}$ & $99.26 \pm 0.13 \mathrm{ab} \mathrm{A}$ \\
\hline \multicolumn{3}{|c|}{ BHT } & $74.69 \pm 0.79 \mathrm{cD}$ & $86.34 \pm 0.84 \mathrm{cC}$ & $90.47 \pm 0.62 \mathrm{c} \mathrm{B}$ & $95.32 \pm 0.62 \mathrm{cA}$ \\
\hline \multicolumn{3}{|c|}{ BHA } & $7.23 \pm 0.27$ g D & $15.14 \pm 0.51 \mathrm{fC}$ & $51.70 \pm 0.45 \mathrm{~d} \mathrm{~B}$ & $95.72 \pm 0.42 \mathrm{cA}$ \\
\hline \multicolumn{3}{|c|}{ AA } & $95.12 \pm 0.54 \mathrm{~b} \mathrm{~B}$ & $95.23 \pm 0.17 \mathrm{bB}$ & $99.30 \pm 0.08 \mathrm{a} \mathrm{A}$ & $99.87 \pm 0.01 \mathrm{a} \mathrm{A}$ \\
\hline \multirow{6}{*}{ 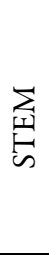 } & \multirow{2}{*}{ ऽ } & Male & $69.46 \pm 0.59 \mathrm{~d} \mathrm{~B}$ & $99.07 \pm 0.15 \mathrm{aA}$ & - & - \\
\hline & & Female & $72.87 \pm 0.47 \mathrm{c} \mathrm{B}$ & $98.89 \pm 0.10 \mathrm{aA}$ & - & - \\
\hline & \multirow{4}{*}{ 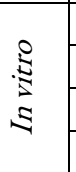 } & Control & $71.77 \pm 0.65 \mathrm{~cd} \mathrm{C}$ & $98.70 \pm 0.14 \mathrm{aB}$ & $99.12 \pm 0.16 \mathrm{ab} \mathrm{A}$ & - \\
\hline & & $50 \mathrm{NaCl}$ & $87.26 \pm 1.07 \mathrm{~b} \mathrm{~B}$ & $99.29 \pm 0.16 \mathrm{aA}$ & $99.81 \pm 0.05$ a A & - \\
\hline & & $100 \mathrm{NaCl}$ & $40.77 \pm 0.44$ e C & $79.70 \pm 0.97 \mathrm{~dB}$ & $98.15 \pm 0.23 \mathrm{~b} \mathrm{~A}$ & - \\
\hline & & $200 \mathrm{NaCl}$ & $34.31 \pm 0.63 \mathrm{fC}$ & $64.39 \pm 1.48 \mathrm{eB}$ & $97.97 \pm 0.39 \mathrm{~b} \mathrm{~A}$ & - \\
\hline \multicolumn{3}{|c|}{ BHT } & $74.69 \pm 0.79 \mathrm{cD}$ & $86.34 \pm 0.84 \mathrm{cC}$ & $90.47 \pm 0.62 \mathrm{cB}$ & $95.32 \pm 0.07 \mathrm{~b} \mathrm{~A}$ \\
\hline \multicolumn{3}{|c|}{$\mathrm{BHA}$} & $7.23 \pm 0.27 \mathrm{~g} \mathrm{D}$ & $15.14 \pm 0.51 \mathrm{fC}$ & $51.70 \pm 0.45 \mathrm{~d} \mathrm{~B}$ & $95.72 \pm 0.09 \mathrm{~b} \mathrm{~A}$ \\
\hline \multicolumn{3}{|c|}{$\mathrm{AA}$} & $95.12 \pm 0.54 \mathrm{a} \mathrm{C}$ & $95.23 \pm 0.17 \mathrm{bB}$ & $99.30 \pm 0.08 \mathrm{ab} \mathrm{A}$ & $99.87 \pm 0.77 \mathrm{a} \mathrm{A}$ \\
\hline \multirow{6}{*}{ 崖 } & \multirow{2}{*}{ ฐ } & Male & $99.26 \pm 0.06 \mathrm{a} \mathrm{A}$ & $99.26 \pm 0.17 \mathrm{aA}$ & - & - \\
\hline & & Female & $99.26 \pm 0.17$ a A & $99.07 \pm 0.09 \mathrm{aA}$ & - & - \\
\hline & \multirow{4}{*}{ 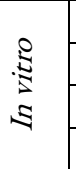 } & Control & $69.00 \pm 0.73 \mathrm{~d} \mathrm{~B}$ & $98.89 \pm 0.27 \mathrm{aA}$ & $98.52 \pm 0.29 \mathrm{a} \mathrm{A}$ & - \\
\hline & & $50 \mathrm{NaCl}$ & $98.70 \pm 0.07$ a A & $99.26 \pm 0.13 \mathrm{aA}$ & - & - \\
\hline & & $100 \mathrm{NaCl}$ & $66.23 \pm 0.82 \mathrm{~dB}$ & $99.26 \pm 0.07 \mathrm{aA}$ & - & - \\
\hline & & $200 \mathrm{NaCl}$ & $40.95 \pm 1.17 \mathrm{fC}$ & $84.87 \pm 0.44 \mathrm{cB}$ & $97.04 \pm 0.29 \mathrm{~b} \mathrm{~A}$ & - \\
\hline \multicolumn{3}{|c|}{ BHT } & $74.69 \pm 0.79 \mathrm{cD}$ & $86.34 \pm 0.84 \mathrm{cC}$ & $90.47 \pm 0.62 \mathrm{c} \mathrm{B}$ & $95.32 \pm 0.07 \mathrm{~b} \mathrm{~A}$ \\
\hline \multicolumn{3}{|c|}{ BHA } & $7.23 \pm 0.27 \mathrm{~g} \mathrm{D}$ & $15.14 \pm 0.51 \mathrm{~d} \mathrm{C}$ & $51.70 \pm 0.45 \mathrm{~d} \mathrm{~B}$ & $95.72 \pm 0.09 \mathrm{~b} \mathrm{~A}$ \\
\hline \multicolumn{3}{|c|}{$\mathrm{AA}$} & $95.12 \pm 0.54 \mathrm{~b} \mathrm{~B}$ & $95.23 \pm 0.17 \mathrm{bB}$ & $99.30 \pm 0.08$ a A & $99.87 \pm 0.77$ a A \\
\hline
\end{tabular}

Each value is the means \pm SE of 3 replicates measurement.

Means followed by different lower-case letters in the columns and upper-case letters in the rows showed significant differences, by the Tukey's test, at $\mathrm{p} \leq 0.05$

Table 3 shows the results of antioxidant activity by the CUPRAC method of in vivo and in vitro samples as absorbance value. Increased absorbance refers to increased activity. Comparing in vivo and in vitro samples, we generally see that in vivo samples exhibit higher activity. Furthermore, in vivo samples showed higher activity than positive controls. In terms of in vitro plants compared to control, the highest values stand out as $1.189 \pm 0.01$ (control $0.143 \pm 0.01$ ) at $100 \mu \mathrm{g} / \mathrm{ml} 200 \mathrm{mM} \mathrm{NaCl}$ in root; $3.117 \pm 0.05$ (control $1.199 \pm 0.02$ ) at $100 \mu \mathrm{g} / \mathrm{ml} 50 \mathrm{mM} \mathrm{NaCl}$ in stem and $1.706 \pm 0.01$ (control $1.029 \pm 0.02$ ) at $50 \mu \mathrm{g} / \mathrm{ml} 50 \mathrm{mM} \mathrm{NaCl}$ in leaf. The activity order of the in vitro samples was found to be leaf> stem> root in parallel with DPPH method findings. 
Ayaz Tilkat E et al. (2020). Not Bot Horti Agrobo 48(4):1885-1900

Table 3. Results of antioxidant activity by CUPRAC method (Absorbance)

\begin{tabular}{|c|c|c|c|c|c|c|}
\hline \multicolumn{3}{|c|}{ Explant type } & $10 \mu \mathrm{g} / \mathrm{ml}$ & $25 \mu \mathrm{g} / \mathrm{ml}$ & $50 \mu \mathrm{g} / \mathrm{ml}$ & $100 \mu \mathrm{g} / \mathrm{ml}$ \\
\hline \multirow{6}{*}{\begin{tabular}{l}
0 \\
0 \\
\hdashline \\
$\simeq 1$
\end{tabular}} & \multirow{2}{*}{$\begin{array}{l}\stackrel{0}{3} \\
\mathbf{s} \\
\mathbf{s}\end{array}$} & Male & $0.835 \pm 0.02 \mathrm{~b} \mathrm{D}$ & $2.058 \pm 0.03 \mathrm{abC}$ & $3.542 \pm 0.02 \mathrm{a} \mathrm{B}$ & $>4$ a A \\
\hline & & Female & $0.835 \pm 0.01 \mathrm{~b} \mathrm{D}$ & $1.907 \pm 0.03 \mathrm{bc} \mathrm{C}$ & $3.394 \pm 0.04 \mathrm{ab} \mathrm{B}$ & $>4 \mathrm{aA}$ \\
\hline & \multirow{4}{*}{$\begin{array}{l}\stackrel{D}{\Xi} \\
\stackrel{\Delta}{s}\end{array}$} & Control & $0.048 \pm 0.01 \mathrm{~d} \mathrm{D}$ & $0.062 \pm 0.01 \mathrm{e} \mathrm{C}$ & $0.100 \pm 0.01 \mathrm{~g} \mathrm{~B}$ & $0.143 \pm 0.01 \mathrm{fA}$ \\
\hline & & $50 \mathrm{NaCl}$ & $0.064 \pm 0.01 \mathrm{dD}$ & $0.084 \pm 0.01$ е C & $0.125 \pm 0.01 \mathrm{~g} \mathrm{~B}$ & $0.171 \pm 0.01 \mathrm{fA}$ \\
\hline & & $100 \mathrm{NaCl}$ & $0.129 \pm 0.02 \mathrm{dD}$ & $0.260 \pm 0.01 \mathrm{~d} \mathrm{C}$ & $0.424 \pm 0.01 \mathrm{fB}$ & $0.520 \pm 0.03$ e A \\
\hline & & $200 \mathrm{NaCl}$ & $0.124 \pm 0.01 \mathrm{dD}$ & $0.309 \pm 0.01 \mathrm{~d} \mathrm{C}$ & $0.604 \pm 0.01 \mathrm{~d} \mathrm{~B}$ & $1.189 \pm 0.01 \mathrm{~d} \mathrm{~A}$ \\
\hline \multicolumn{3}{|c|}{$\mathrm{BHT}$} & $0.90 \pm 0.02 \mathrm{ab} \mathrm{D}$ & $1.75 \pm 0.07$ c C & $2.33 \pm 0.05 \mathrm{c} \mathrm{B}$ & $2.6 \pm 0.02 \mathrm{c} \mathrm{A}$ \\
\hline \multicolumn{3}{|c|}{$\mathrm{BHA}$} & $0.96 \pm 0.04 \mathrm{a} \mathrm{D}$ & $2.20 \pm 0.05 \mathrm{aC}$ & $3.30 \pm 0.03 \mathrm{~b} \mathrm{~B}$ & $3.65 \pm 0.05 \mathrm{~b} \mathrm{~A}$ \\
\hline \multicolumn{3}{|c|}{$\mathrm{AA}$} & $0.32 \pm 0.02 \mathrm{cD}$ & $1.78 \pm 0.03 \mathrm{c} \mathrm{C}$ & $3.52 \pm 0.05 \mathrm{a} \mathrm{B}$ & $3.70 \pm 0.04 \mathrm{~b} \mathrm{~A}$ \\
\hline \multirow{6}{*}{$\underset{⿱ ㇒}{\sum_{\infty}}$} & \multirow{2}{*}{$\Xi \stackrel{8}{s}$} & Male & $0.407 \pm 0.01 \mathrm{~b} \mathrm{D}$ & $0.846 \pm 0.01$ с C & $1.714 \pm 0.01 \mathrm{~d} \mathrm{~B}$ & $3.773 \pm 0.02$ a A \\
\hline & & Female & $0.301 \pm 0.01 \mathrm{~d} \mathrm{D}$ & $0.610 \pm 0.01 \mathrm{de} C$ & $1.197 \pm 0.01 \mathrm{fB}$ & $2.353 \pm 0.02 \mathrm{~d} \mathrm{~A}$ \\
\hline & \multirow{4}{*}{$\begin{array}{l}\stackrel{D}{\Xi} \\
\Sigma\end{array}$} & Control & $0.261 \pm 0.01 \mathrm{dD}$ & $0.566 \pm 0.01 \mathrm{e} \mathrm{C}$ & $1.075 \pm 0.01 \mathrm{fB}$ & $1.199 \pm 0.02 \mathrm{e} \mathrm{A}$ \\
\hline & & $50 \mathrm{NaCl}$ & $0.396 \pm 0.02 \mathrm{bc} D$ & $0.742 \pm 0.01 \mathrm{~cd} \mathrm{C}$ & $1.438 \pm 0.01 \mathrm{e} \mathrm{B}$ & $3.117 \pm 0.05 \mathrm{~b} \mathrm{~A}$ \\
\hline & & $100 \mathrm{NaCl}$ & $0.152 \pm 0.01$ e D & $0.284 \pm 0.01 \mathrm{fC}$ & $0.525 \pm 0.01 \mathrm{~g} \mathrm{~B}$ & $1.126 \pm 0.03 \mathrm{e} \mathrm{A}$ \\
\hline & & $200 \mathrm{NaCl}$ & $0.142 \pm 0.01$ e D & $0.278 \pm 0.01 \mathrm{fC}$ & $0.472 \pm 0.01 \mathrm{~g} \mathrm{~B}$ & $0.498 \pm 0.01 \mathrm{fA}$ \\
\hline \multicolumn{3}{|c|}{ BHT } & $0.90 \pm 0.02 \mathrm{a} \mathrm{D}$ & $1.75 \pm 0.07 \mathrm{~b} \mathrm{C}$ & $2.33 \pm 0.05 \mathrm{c} \mathrm{B}$ & $2.6 \pm 0.02 \mathrm{c} \mathrm{A}$ \\
\hline \multicolumn{3}{|c|}{$\mathrm{BHA}$} & $0.96 \pm 0.04$ a D & $2.20 \pm 0.05 \mathrm{a} \mathrm{C}$ & $3.30 \pm 0.03 \mathrm{~b} \mathrm{~B}$ & $3.65 \pm 0.05$ a A \\
\hline \multicolumn{3}{|c|}{ AA } & $0.32 \pm 0.02 \mathrm{~cd} \mathrm{D}$ & $1.78 \pm 0.03 \mathrm{~b} \mathrm{C}$ & $3.52 \pm 0.05 \mathrm{a} \mathrm{B}$ & $3.70 \pm 0.04 \mathrm{a} \mathrm{A}$ \\
\hline \multirow{6}{*}{$\begin{array}{l}\text { 岸 } \\
\text { 夏 }\end{array}$} & \multirow{2}{*}{ ฐ } & Male & $0.517 \pm 0.01 \mathrm{c} \mathrm{D}$ & $1.195 \pm 0.01 \mathrm{~d} \mathrm{C}$ & $2.351 \pm 0.01 \mathrm{~dB}$ & $>4$ a A \\
\hline & & Female & $0.648 \pm 0.01 \mathrm{~b} \mathrm{D}$ & $1.472 \pm 0.01 \mathrm{c} \mathrm{C}$ & $2.837 \pm 0.02 \mathrm{c} \mathrm{B}$ & $>4$ a A \\
\hline & \multirow{4}{*}{$\begin{array}{l}0 \\
\text { : } \\
\text { s }\end{array}$} & Control & $0.224 \pm 0.01 \mathrm{fD}$ & $0.505 \pm 0.01 \mathrm{fC}$ & $1.029 \pm 0.02 \mathrm{fB}$ & $2.00 \pm 0.03 \mathrm{~d} \mathrm{~A}$ \\
\hline & & $50 \mathrm{NaCl}$ & $0.387 \pm 0.01 \mathrm{~d} \mathrm{D}$ & $0.85 \pm 0.01$ e C & $1.706 \pm 0.01 \mathrm{e} \mathrm{B}$ & $>4$ a A \\
\hline & & $100 \mathrm{NaCl}$ & $0.293 \pm 0.01$ ef D & $0.542 \pm 0.01 \mathrm{fC}$ & $1.021 \pm 0.01 \mathrm{fB}$ & $2.045 \pm 0.02 \mathrm{dA}$ \\
\hline & & $200 \mathrm{NaCl}$ & $0.123 \pm 0.01 \mathrm{~g} \mathrm{D}$ & $0.255 \pm 0.01 \mathrm{~g} \mathrm{C}$ & $0.476 \pm 0.01 \mathrm{~g} \mathrm{~B}$ & $1.062 \pm 0.01 \mathrm{e} \mathrm{A}$ \\
\hline \multicolumn{3}{|c|}{$\mathrm{BHT}$} & $0.90 \pm 0.02 \mathrm{a} D$ & $1.75 \pm 0.07 \mathrm{~b} \mathrm{C}$ & $2.33 \pm 0.05 \mathrm{~dB}$ & $2.6 \pm 0.02 \mathrm{c} \mathrm{A}$ \\
\hline \multicolumn{3}{|c|}{$\mathrm{BHA}$} & $0.96 \pm 0.04$ a D & $2.20 \pm 0.05 \mathrm{a} \mathrm{C}$ & $3.30 \pm 0.03 \mathrm{~b} \mathrm{~B}$ & $3.65 \pm 0.05 \mathrm{~b} \mathrm{~A}$ \\
\hline \multicolumn{3}{|c|}{ AA } & $0.32 \pm 0.02 \mathrm{de} \mathrm{D}$ & $1.78 \pm 0.03 \mathrm{~b} \mathrm{C}$ & $3.52 \pm 0.05 \mathrm{aB}$ & $3.70 \pm 0.04 \mathrm{~b} \mathrm{~A}$ \\
\hline
\end{tabular}

Each value is the means \pm SE of 3 replicates measurement.

Means followed by different lower-case letters in the columns and uppercase letters in the rows showed significant differences by the Tukey's test, at $\mathrm{p} \leq 0.05$

As we have seen in Table 4, total phenolic and flavonoid content results of all samples are given as equivalent to gallic acid and quercetin, respectively. According to Table 4, in vivo samples showed higher phenolic content than in vitro samples, whereas all samples showed similar values in terms of flavonoids. The activity and total phenolic content order in the in vivo samples was found to be as root>leaf $>$ stem; and in the in vitro samples, leaf > stem> root. Regarding the total phenolic and flavonoid content, our results showed significantly higher values in in vivo samples as compared to in vitro samples.

In vitro samples were found to respond differently depending on the concentration of salt applied. Increased activity and increased phenolic content were determined depending on increased salt concentration in root samples. This phenomenon is not obtained for stem and leaf samples. While the activity and phenolic content increased at $50 \mathrm{mM} \mathrm{NaCl}$ concentration compared to the control, then a decrease in activity was detected according to increasing salt concentration. Many studies are indicating the changes in phenolic compounds amount of plants under salt stress (Yuan et al., 2010; Falleh et al., 2012; Arzani and Ashraf, 2016; Golkar and Taghizadeh, 2018; Golkar et al., 2019).

To evaluate the results of the chemical analysis as a summary; in vivo plant extracts were determined to have higher chemical activity compared to extracts from different parts of plants germinated in MS medium with low or no salt content. However, it has been determined that when the amount of extract and salt concentration is increased, in vitro extracts may exhibit higher chemical activity than the control extracts. 
Table 4. Total phenolic and flavonoid content ${ }^{\mathrm{a}}$

\begin{tabular}{|c|c|c|c|c|}
\hline \multicolumn{3}{|c|}{ Explant type } & Total phenolic content & Total flavonoid content \\
\hline \multirow{6}{*}{\begin{tabular}{l}
$\stackrel{5}{0}$ \\
\hdashline \\
\hdashline
\end{tabular}} & \multirow{2}{*}{$\begin{array}{l}\mathrm{S} \\
\mathrm{S} \\
\mathrm{S}\end{array}$} & Male & $492.55 \pm 1.02 \mathrm{a}$ & $45.47 \pm 0.45 a$ \\
\hline & & Female & $471.48 \pm 0.79 b$ & $42.24 \pm 0.47 b$ \\
\hline & \multirow{4}{*}{$\begin{array}{l}\mathbb{Z} \\
\mathbb{S}\end{array}$} & Control & $57.06 \pm 0.48 \mathrm{f}$ & $32.40 \pm 0.35 c$ \\
\hline & & $50 \mathrm{NaCl}$ & $69.80 \pm 0.73 \mathrm{e}$ & $34.16 \pm 0.57 c$ \\
\hline & & $100 \mathrm{NaCl}$ & $92.27 \pm 1.13 \mathrm{~d}$ & $33.36 \pm 0.57 \mathrm{c}$ \\
\hline & & $200 \mathrm{NaCl}$ & $99.29 \pm 0.49 \mathrm{c}$ & $34.16 \pm 0.51 \mathrm{c}$ \\
\hline \multirow{6}{*}{$\underset{\text { 剀 }}{\sum_{\infty}}$} & \multirow{2}{*}{$\begin{array}{l}0 \\
5 \\
5\end{array}$} & Male & $236.93 \pm 1.00 \mathrm{a}$ & $41.43 \pm 0.73 b$ \\
\hline & & Female & $175.14 \pm 1.28 \mathrm{c}$ & $36.59 \pm 0.41 c$ \\
\hline & \multirow{4}{*}{$\begin{array}{l}: \\
\mathbb{S} \\
\mathbb{S}\end{array}$} & Control & $145.64 \pm 0.85 \mathrm{~d}$ & $42.24 \pm 0.82 b$ \\
\hline & & $50 \mathrm{NaCl}$ & $181.72 \pm 0.81 \mathrm{~b}$ & $45.18 \pm 0.68 a$ \\
\hline & & $100 \mathrm{NaCl}$ & $103.51 \pm 0.36 \mathrm{e}$ & $42.24 \pm 0.35 b$ \\
\hline & & $200 \mathrm{NaCl}$ & $97.89 \pm 0.58 \mathrm{f}$ & $34.97 \pm 0.33 c$ \\
\hline \multirow{6}{*}{ 崖 } & \multirow{2}{*}{$\begin{array}{l}8 \\
s \\
s\end{array}$} & Male & $284.69 \pm 0.95 b$ & $40.63 \pm 0.82 b c$ \\
\hline & & Female & $342.27 \pm 1.88 \mathrm{a}$ & $54.36 \pm 0.62 a$ \\
\hline & \multirow{4}{*}{$\begin{array}{l}\mathbb{N} \\
\mathbb{S} \\
\mathbb{S}\end{array}$} & Control & $141.43 \pm 0.47 \mathrm{~d}$ & $54.36 \pm 0.51 \mathrm{a}$ \\
\hline & & $50 \mathrm{NaCl}$ & $196.20 \pm 0.87 \mathrm{c}$ & $39.01 \pm 0.30 c$ \\
\hline & & $100 \mathrm{NaCl}$ & $142.83 \pm 1.87 \mathrm{~d}$ & $42.24 \pm 0.29 b$ \\
\hline & & $200 \mathrm{NaCl}$ & $86.65 \pm 1.04 \mathrm{e}$ & $35.78 \pm 0.64 \mathrm{~d}$ \\
\hline
\end{tabular}

Each value is the means \pm SE of 3 replicates measurement.

Means followed by different lower-case letters in the columns showed significant differences, by the Tukey's test, at $\mathrm{p} \leq 0.05$

a: GAs, gallic acid equivalents $(y=0.0356 x-0.0047 \mathrm{R} 2=0.997)$

b: QEs, quercetin equivalents $(\mathrm{y}=0.0619 \mathrm{x}-0.0043 \mathrm{R} 2=0.9991)$

Table 5. shows the antimicrobial activities of the in vivo and in vitro plant extracts. Inactive samples are not included in the table. The in vivo plant extracts found to be active in terms of antimicrobial effect, especially, female parts are more active than male parts. The inhibition zone diameters of in vivo samples range from $8 \mathrm{~mm}$ to $16 \mathrm{~mm}$, and the MIC values from $100 \mu \mathrm{g} / \mathrm{ml}$ to $800 \mu \mathrm{g} / \mathrm{ml}$. The best activity was $16 \mathrm{~mm}$ inhibition zone diameter and $100 \mu \mathrm{g} / \mathrm{ml}$ MIC value recorded by female leaf extract against C. albicans and by male root extract against $P$. aeruginosa.

We could not see the same activity in in vitro samples. Most of the in vitro samples did not exhibit any activity. However, $100 \mathrm{mM} \mathrm{NaCl}$ leaf extract exhibited moderate antimicrobial activity (inhibition zone diameter between $12-20 \mathrm{~mm}$ ) against $S$. aureus $(17 \pm 0.6$ zone diameter, $50 \pm 4.0 \mathrm{MIC}$ value) and $C$. albicans $(15 \pm 0.0$ zone diameter, $100 \pm 5.0 \mathrm{MIC}$ value). While antimicrobial activity was not observed in root control, 100 and $200 \mathrm{mM} \mathrm{NaCl}$ applications induced the activity against $E$. coliand $P$. aeruginosa. Similarly, we see that 50,100 and $200 \mathrm{mM} \mathrm{NaCl}$ applications on the leaf increase the antimicrobial activity compared to control. 
Table 5. Inhibition zone diameters and MIC values of in vivo and in vitro samples

\begin{tabular}{|c|c|c|c|c|c|c|c|c|}
\hline \multicolumn{3}{|c|}{ Explant type } & & E. coli & P. aeruginosa & S. aureus & S. pyogenes & C. albicans \\
\hline \multirow{4}{*}{$\begin{array}{l}\stackrel{1}{0} \\
\stackrel{1}{\simeq}\end{array}$} & \multirow{2}{*}{$\begin{array}{l}\stackrel{0}{s} \\
\text { s }\end{array}$} & Male & $\begin{array}{c}\mathrm{DD} \\
\mathrm{MIC}\end{array}$ & $\begin{array}{c}9 \pm 0.3 \mathrm{c} \\
500 \pm 6.0 \mathrm{~B}\end{array}$ & $\begin{array}{c}16 \pm 3.0 \mathrm{a} \\
100 \pm 0.11 \mathrm{C}\end{array}$ & $\begin{array}{c}9 \pm 0.6 \mathrm{c} \\
500 \pm 17.0 \mathrm{~A}\end{array}$ & $\begin{array}{c}10 \pm 0.0 \mathrm{~b} \\
500 \pm 12 \mathrm{~B}\end{array}$ & $\begin{array}{l}13 \pm 0.3 \mathrm{c} \\
200 \pm 5 \mathrm{~A}\end{array}$ \\
\hline & & Female & $\begin{array}{c}\mathrm{DD} \\
\mathrm{MIC}\end{array}$ & $\begin{array}{c}15 \pm 0.9 b \\
100 \pm 3.0 \mathrm{C}\end{array}$ & $\begin{array}{l}13 \pm 0.60 \mathrm{~b} \\
200 \pm 2.0 \mathrm{~B}\end{array}$ & $\begin{array}{c}12 \pm 0.6 \mathrm{~b} \\
400 \pm 17.0 \mathrm{~B}\end{array}$ & $\begin{array}{c}10 \pm 0.1 \mathrm{~b} \\
500 \pm 0.14 \mathrm{~B}\end{array}$ & $\begin{array}{l}14 \pm 0.2 \mathrm{~b} \\
200 \pm 1 \mathrm{~A}\end{array}$ \\
\hline & \multirow{2}{*}{ 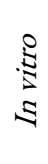 } & $100 \mathrm{NaCl}$ & $\begin{array}{l}\text { DD } \\
\text { MIC }\end{array}$ & $\begin{array}{c}7.66 \pm 0.3 \mathrm{c} \\
>1000 \mathrm{~A}\end{array}$ & $\begin{array}{l}7 \pm 0.00 \mathrm{c} \\
>1000 \mathrm{~A}\end{array}$ & $\begin{array}{c}\mathrm{NZ} \\
-\end{array}$ & $\begin{array}{c}7 \pm 0 \mathrm{c} \\
>1000 \mathrm{~A}\end{array}$ & $\begin{array}{c}\mathrm{NZ} \\
-\end{array}$ \\
\hline & & $200 \mathrm{NaCl}$ & $\begin{array}{c}\mathrm{DD} \\
\mathrm{MIC}\end{array}$ & $\begin{array}{c}7 \pm 0 \mathrm{c} \\
>1000 \mathrm{~A}\end{array}$ & $\begin{array}{c}\mathrm{NZ} \\
-\end{array}$ & $\begin{array}{c}\mathrm{NZ} \\
-\end{array}$ & $\begin{array}{c}\mathrm{NZ} \\
-\end{array}$ & $\begin{array}{c}\mathrm{NZ} \\
-\end{array}$ \\
\hline \multicolumn{3}{|c|}{ PC } & $\begin{array}{l}\text { DD } \\
\text { MIC }\end{array}$ & $\begin{array}{c}20 \pm 0.6 \mathrm{a} \\
7.815 \pm 0.1 \mathrm{D}\end{array}$ & $\begin{array}{c}\mathrm{NZ} \\
-\end{array}$ & $\begin{array}{c}35 \pm 0.0 \mathrm{a} \\
1.95 \pm 0.3 \mathrm{C}\end{array}$ & $\begin{array}{c}19 \pm 0.1 \mathrm{a} \\
7.815 \pm 0.2 \mathrm{C}\end{array}$ & $\begin{array}{c}30 \pm 0.0 \mathrm{a} \\
3.125 \pm 0.1 \mathrm{~B}\end{array}$ \\
\hline \multirow{2}{*}{$\sum_{\text {出 }}$} & \multirow{2}{*}{ 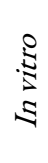 } & Male & $\begin{array}{l}\mathrm{DD} \\
\mathrm{MIC} \\
\end{array}$ & $\begin{array}{r}10 \pm 0.3 b \\
500 \pm 10 \mathrm{~A} \\
\end{array}$ & $\begin{array}{c}9 \pm 0.6 \mathrm{~b} \\
500 \pm 16 \mathrm{~A}\end{array}$ & $\begin{array}{l}10 \pm 0.0 \mathrm{~b} \\
500 \pm 6 \mathrm{~A} \\
\end{array}$ & $\begin{array}{c}9 \pm 0.2 \mathrm{~b} \\
500 \pm 0.0 \mathrm{~B}\end{array}$ & $\begin{array}{l}7 \pm 0.0 \mathrm{c} \\
>1000 \mathrm{~A} \\
\end{array}$ \\
\hline & & Female & $\begin{array}{c}\mathrm{DD} \\
\mathrm{MIC}\end{array}$ & $\begin{array}{c}10 \pm 0.5 \mathrm{~b} \\
500 \pm 29 \mathrm{~A}\end{array}$ & $\begin{array}{c}15.03 \pm 0.4 \mathrm{a} \\
100 \pm 3 \mathrm{~B}\end{array}$ & $\begin{array}{c}9 \pm 0.2 \mathrm{c} \\
500 \pm 10 \mathrm{~A}\end{array}$ & $\begin{array}{c}7.83 \pm 0.2 \mathrm{c} \\
800 \pm 6 \mathrm{~A}\end{array}$ & $\begin{array}{c}13 \pm 0.6 \mathrm{~b} \\
200 \pm 1.0 \mathrm{~B}\end{array}$ \\
\hline \multicolumn{3}{|c|}{ PC } & $\begin{array}{c}\mathrm{DD} \\
\mathrm{MIC}\end{array}$ & $\begin{array}{c}20 \pm 0.6 \mathrm{a} \\
7.815 \pm 0.1 \mathrm{~B}\end{array}$ & $\begin{array}{c}\mathrm{NZ} \\
-\end{array}$ & $\begin{array}{c}35 \pm 0.0 \mathrm{a} \\
1.95 \pm 0.3 \mathrm{~B}\end{array}$ & $\begin{array}{c}19 \pm 0.6 \mathrm{a} \\
7.815 \pm 0.2 \mathrm{C}\end{array}$ & $\begin{array}{c}30 \pm 0.0 \mathrm{a} \\
3.125 \pm 0.1 \mathrm{C}\end{array}$ \\
\hline \multirow{6}{*}{ 崖 } & \multirow{2}{*}{$\begin{array}{l}\frac{5}{5} \\
\frac{5}{5}\end{array}$} & Male & $\begin{array}{c}\mathrm{DD} \\
\mathrm{MIC}\end{array}$ & $\begin{array}{c}9 \pm 0.00 \mathrm{c} \\
500 \pm 20.0 \mathrm{~B}\end{array}$ & $\begin{array}{l}13.03 \pm 0.4 \mathrm{a} \\
200 \pm 8.0 \mathrm{D}\end{array}$ & $\begin{array}{c}12 \pm 0.6 \mathrm{c} \\
400 \pm 7.0 \mathrm{~A}\end{array}$ & $\begin{array}{c}11 \pm 0.3 \mathrm{c} \\
400 \pm 2.0 \mathrm{~B}\end{array}$ & $\begin{array}{c}9 \pm 0.0 \mathrm{e} \\
500 \pm 3.0 \mathrm{~A}\end{array}$ \\
\hline & & Female & $\begin{array}{l}\text { DD } \\
\text { MIC }\end{array}$ & $\begin{array}{c}13 \pm 0.6 b \\
200 \pm 8.0 \mathrm{C}\end{array}$ & $\begin{array}{c}10 \pm 0.3 \mathrm{c} \\
500 \pm 0.0 \mathrm{~B}\end{array}$ & $\begin{array}{c}13 \pm 0.6 \mathrm{c} \\
200 \pm 9.0 \mathrm{~B}\end{array}$ & $\begin{array}{c}15.03 \pm 0.4 \mathrm{~b} \\
100 \pm 6.0 \mathrm{C}\end{array}$ & $\begin{array}{c}16 \pm 0.0 \mathrm{~b} \\
100 \pm 5.0 \mathrm{~B}\end{array}$ \\
\hline & \multirow{4}{*}{ 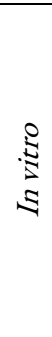 } & Control & $\begin{array}{c}\mathrm{DD} \\
\mathrm{MIC}\end{array}$ & $\begin{array}{c}\mathrm{NZ} \\
-\end{array}$ & $\begin{array}{c}7 \pm 0.00 \mathrm{~d} \\
>1000 \mathrm{~A}\end{array}$ & $\begin{array}{c}\mathrm{NZ} \\
-\end{array}$ & $\begin{array}{c}\mathrm{NZ} \\
-\end{array}$ & $\begin{array}{c}\mathrm{NZ} \\
-\end{array}$ \\
\hline & & $50 \mathrm{NaCl}$ & $\begin{array}{c}\mathrm{DD} \\
\mathrm{MIC}\end{array}$ & $\begin{array}{c}9 \pm 0.2 \mathrm{c} \\
500 \pm 18.0 \mathrm{~B}\end{array}$ & $\begin{array}{c}10 \pm 0.1 \mathrm{c} \\
500 \pm 2.0 \mathrm{~B}\end{array}$ & $\begin{array}{c}12 \pm 0.3 \mathrm{c} \\
400 \pm 6.0 \mathrm{~A}\end{array}$ & $\begin{array}{c}9 \pm 0.0 \mathrm{~d} \\
500 \pm 12.0 \mathrm{~A}\end{array}$ & $\begin{array}{c}10 \pm 0.4 \mathrm{~d} \\
500 \pm 0.19 \mathrm{~A}\end{array}$ \\
\hline & & $100 \mathrm{NaCl}$ & $\begin{array}{c}\mathrm{DD} \\
\mathrm{MIC}\end{array}$ & $\begin{array}{c}10 \pm 0.2 \mathrm{c} \\
500 \pm 10.0 \mathrm{~B}\end{array}$ & $\begin{array}{l}11 \pm 0.57 b \\
400 \pm 5.2 \mathrm{C}\end{array}$ & $\begin{array}{l}17 \pm 0.6 \mathrm{~b} \\
50 \pm 4.0 \mathrm{C}\end{array}$ & $\begin{array}{c}12 \pm 0.6 \mathrm{c} \\
400 \pm 5.0 \mathrm{~B} \\
\end{array}$ & $\begin{array}{c}15 \pm 0.0 \mathrm{c} \\
100 \pm 2.0 \mathrm{~B} \\
\end{array}$ \\
\hline & & $200 \mathrm{NaCl}$ & $\begin{array}{l}\text { DD } \\
\text { MIC }\end{array}$ & $\begin{array}{c}7 \pm 0.00 \mathrm{~d} \\
>1000 \mathrm{~A} \\
\end{array}$ & $\begin{array}{c}\mathrm{NZ} \\
- \\
\end{array}$ & $\begin{array}{c}\text { NZ } \\
- \\
\end{array}$ & $\begin{array}{c}\text { NZ } \\
- \\
\end{array}$ & $\begin{array}{c}\text { NZ } \\
- \\
\end{array}$ \\
\hline & & $\mathrm{C}$ & $\begin{array}{l}\mathrm{DD} \\
\mathrm{MIC}\end{array}$ & $\begin{array}{c}20 \pm 0.6 \mathrm{a} \\
7.815 \pm 0.12 \\
\mathrm{D}\end{array}$ & $\begin{array}{c}\mathrm{NZ} \\
-\end{array}$ & $\begin{array}{c}35 \pm 0.0 \mathrm{a} \\
1.95 \pm 0.3 \mathrm{D}\end{array}$ & $\begin{array}{c}19 \pm 0.6 \mathrm{a} \\
7.815 \pm 0.2 \mathrm{D}\end{array}$ & $\begin{array}{c}30 \pm 0.0 \mathrm{a} \\
3.125 \pm 0.07 \\
\mathrm{C}\end{array}$ \\
\hline
\end{tabular}

Each value is the means \pm SE of 3 replicates measurement. Means followed by different lower-case letters in the columns for DD and upper-case letters in the columns for MIC showed significant differences, by the Tukey's test, at $\mathrm{p} \leq 0.05$

Each disc contains $1.5 \mathrm{mg}$ extract. DD: disc diameter in $\mathrm{mm}$. MIC: minimum inhibitory concentration in $\mu \mathrm{g} / \mathrm{ml}$. NZ: No zone C: control of in vitroleaf. PC: positive control (ampicillin and nystatin).

\section{Discussion}

Under stress conditions, including salinity, the speed of reactive oxygen species (ROS) will increase, and the resulting stress can cause harmful oxidation of various plant components. To prevent oxidation, ROS concentration in the plant cells is kept in check by several scavenging antioxidant compounds (Apel and Hirt, 2004).

Various adaptation tolls have been developed by higher plants for extreme conditions through cascade biosynthesis for both enzymatic and non-enzymatic antioxidants to protect themselves against ROS production by detoxification systems under salinity stress (Arzani and Ashraf, 2016; Golkar and Taghizadeh, 2018). Phenolic compounds are among such antioxidants, and several studies have reported changes in their concentrations in plants upon salinity stress (Yuan et al., 2010; Falleh et al., 2012; Golkar and Taghizadeh, 
2018; Golkar et al., 2019). This change was observed as both increasing (Ksouri et al., 2007) and decreasing depending on the stress levels (Navarro et al., 2006). Besides, it has been reported that these phenolic components are in different concentrations in different parts of the same plant, with their increases positively correlated with increases in antioxidant activity (Hatamnia et al., 2016; Golkar and Taghizadeh, 2018; Golkar et al., 2019). The results of these previous studies are comparable to those obtained in our current study. Previous research has indicated that plants react to salinity by increasing their antioxidant activity potentials (Hernandez and Almansa, 2002; Falleh et al., 2012; Javed and Gürel, 2019) through the metabolites commonly found in them that demonstrate antioxidant activity and are effective defence mechanisms against oxidative stress from free radicals (Sacchetti et al., 2005). Moreover, the increases in these metabolites also enhance tolerance levels in plants against oxidative stress coming from increases in salinity (Ksouri et al., 2007).

Several different methods were developed to measure the antioxidant capacity, which has a different substrate, function, polarity, source, and mechanism. Besides, the extraction method, parts of the plant, age of the plant, and the plant's environmental conditions are important factors for measuring antioxidant activity (Brewer, 2011; Esmaeili et al., 2015). In this study, three methods by which to measure antioxidant activity in plants 2,2-azino-bis (ethylbenzene-thiazoline-6-sulfonic acid (ABTS), 2,2-diphenyl-1-picrylhydrazyl (DPPH), and cupric ion reducing antioxidant capacity (CUPRAC) were used, the best results of which were obtained from the ABTS measurements.

Pistacia species contains several different bioactive compounds, such as triterpenes, essential oils, and phenolic compounds, and different ratios of these compounds are found in different parts of the plant, such as its resin, stem, leaves, and fruit (Kaliora et al., 2004; Assimopoulou, 2005; Tilkat et al., 2018); consequently, we attributed the differences in antioxidant activities determined from our results to these concentration differences. The overall evaluation of our results indicates that plant samples in vivo demonstrated higher antioxidant activity and phenolic ingredients than their counterparts in vitro. Esmaeili et al. (2015) have examined the total phenolic content (TPC), flavonoid content (TFC), and antioxidant activity in vitro, in vivo, and callus tissue from Trifolium pratense. All TPC, TFC, and antioxidant activities were in the order of in vitro > callus > in vivo. Parsaeimehr et al. (2010) have demonstrated results comparable to those in our in vitro and in vivo studies. Similarly, we have deduced that in vivo samples have higher potential than in vitro samples regarding the total phenolic and flavonoid content, antioxidant and antimicrobial activity.

In the literature, the studies comparing mostly between wild plants and plants grown in an in vitro culture medium are encountered. However, there is no study comparing antioxidant and antimicrobial activity between wild plants and plants treated with salt in vitro up to now. So, this is the first study to be performed in this sense.

Variation in the amount and diversity of secondary plant metabolites under abiotic stress has been reported by several studies (Navarro et al., 2006; Valifard et al., 2014). The antimicrobial activity most probably affiliated to the chemical structure as well as the amount of the most abundant compounds and interaction of major and minor active compounds (Dorman and Deans, 2000; Delaquis et al., 2002; Mazari et al., 2010). Salinity could affect the antimicrobial activity as it has significant effects on the quality and quantity of the different composition of secondary metabolites. According to our results, the antimicrobial activity exhibited diversity with organs type, and salt stress level and these results may be explained by the possible variations of the chemical structure of $P$. khinjuk plant grown under $\mathrm{NaCl}$ stress.

In many studies comparing antimicrobial activities of in vitro and in vivo samples, different results have been obtained indicating that activity increases, decreases or remains constant (Taran et al., 2010; Cuce et al., 2017; Ahmed et al., 2018; Taghizadeh et al., 2018). Contrary to our results, Salem et al. (2014) reported that salinity reduces antimicrobial activity in Carthamus tinctorius plant. When we compare in vivo and in vitro control samples; it is clear that the in vivo samples are more active, while the activity appears to be increased up to $100 \mathrm{mM} \mathrm{NaCl}$ when comparing salt-eluted samples with in vitro control samples. We consider that the activity has decreased as a result of damage in the physiological mechanism by the in vitro elicitation. Briefly, in terms of antimicrobial activity, female plant extracts are more active than all other tested extracts. 
As a result, although increased salinity values significantly reduced antimicrobial activity, it is determined that $100 \mathrm{mM} \mathrm{NaCl}$ applications to in vitro leaf extracts exhibited moderate antimicrobial activity against $S$. aureus and $C$. albicans.

\section{Conclusions}

Our study aimed to reveal the antioxidant and antimicrobial activities of the different plant parts (root, stem and leaf) of the Pistacia khinjuk Stocks plant both in vitro and in vivo, and to determine whether these activities increase or not, especially with salt elicitations.

As a result of our study, it was determined that the antioxidant and antimicrobial activities of all in vivo samples had close or higher activity than the standard BHT, BHA, AA, and in vivo samples had higher activity in terms of both parameters compared to in vitro samples. Changes in activities were found in positive correlation with the increase or decrease in the total amount of phenolic and flavonoid substances. It is clear that salinity can change the metabolic profile of plants, and this metabolic profile change causes them to differ in their antimicrobial and antioxidant activities.

\section{Authors' Contributions}

EAT: In vitro propagation of the plant, $\mathrm{NaCl}$ elicitation to in vitro plants, experimental design and preparation of the manuscript. NH: Antimicrobial studies as well as preparation of the manuscript. İSK: Conducted antioxidant studies and preparation of the manuscript. VS: Conducted the statistical analysis and preparation of manuscript according to journal rules. All authors read and approved the final manuscript.

\section{Acknowledgements}

The study was funded by a grant from BTUBAP-Batman University Research Project Council BTÜBAP-2018-FED-1. We also thank Israt Jahan for linguistically reviewing the manuscript.

\section{Conflict of Interests}

The authors declare that there are no conflicts of interest related to this article.

\section{References}

Ahmed ABA, Magesh J, Idris SN, Mubarak EN, Taha RM (2018). Chemical constituents and antimicrobial activity of essential oils from micropropagation and field grown plants of Wedelia biflora L. Journal of Essential Oil-Bearing Plants 21(6):1712-1724. https://doi.org/10.1080/0972060X.2018.1538819

Ahmed S, Saeed-Ul-Hassan S, Islam M, Qureshi F, Waheed I, Munawar I (2017). Anti-oxidant activity of Pistachia khinjuk supported by phytochemical investigation. Acta Poloniae Pharmaceutica 74:173-78.

Apak R, Güclü K, Özyürek M, Karademir SE (2004). A novel total antioxidant capacity index for dietary polyphenols, vitamin $\mathrm{C}$ and $\mathrm{E}$, using their cupric ion reducing capability in the presence of neocuproine: The CUPRAC method. Journal of Agriculture Food Chemistry 52:7970-7981. https://doi.org/10.1021/jf048741X

Apel K, Hirt H (2004). Reactive oxygen species: Metabolism, oxidative stress and signal transduction. Annual Review of Plant Biology 55:373-399. https://doi.org/10.1146/annurev.arplant.55.031903.141701 
Arzani A, Ashraf, M (2016). Smart engineering of genetic resources for enhanced salinity tolerance in crop plants. Critical Review Plant Science 35:146-189. https://doi.org/10.1080/07352689.2016.1245056

Assimopoulou AN, Papageorgiou VP (2005). GC-MS analysis of penta- and tetra-cyclic triterpenes from resins of Pistacia species. Part II. Pistacia terebinthus var. chia. Biomedical Chromatography 19:586-605. https://doi.org.10.1002/bmc.484

Ayaz Tilkat E, Kaplan A, Hoşer A, Tilkat E (2017). In vitro şartlarda yetiştirilen Buttum (Pistacia khinjuk Stocks)'da çözünür karbonhidrat değerleri ile antioksidan peroksidaz aktivitesi üzerine tuz stresinin etkileri. Batman Üniversitesi Yaşam Bilimleri Dergisi 7(2):90-97. https://hdl.handle.net/20.500.12402/1769

Ayaz Tilkat E, Kaplan A, Tilkat E, Bağlamış G, Onay A (2019). Effects of salt stress on morpho-physiological and biochemical characters of Lentisk (P. lentiscus L.). International Journal of Nature and Life Sciences 3(1):20-31. https://dergipark.org.tr/tr/pub/ijnls/issue/43966/533316

Benmahioul B, Daguin F, Kaid-Harche M (2009) Effects of salt stress on germination and in vitro growth of pistachio (Pistacia vera L.). Comptes Rendus Biologies 332(8):752-8. https://doi.org/10.1016/j.crvi.2009.03.008

Blois MS (1958). Antioxidant determinations by the use of a stable free radical. Nature 181:1199-1200. https://doi.org/10.1038/1811199a0

Brewer MS (2011). Natural antioxidants: Sources, compounds, mechanisms of action, and potential applications. Comprehensive Reviews in Food Science and Food Safety 10:221-247. https://doi.org/10.1111/j.1541-4337.2011.00156.x

Chelli-Chaabouni A, Mosbah AB, Maalej M, Gargouri K, Gargouri-Bouzid R, Drira N (2010). In vitro salinity tolerance of two pistachio rootstocks: Pistacia vera L. and P. atlantica Desf. Environmental and Experimental Botany 69(3):302-312. https://doi.org/10.1016/j.envexpbot.2010.05.010

Cuce M, Bekircan T, Laghari AH, Sökmen M, Sökmen A, Önay Uçar E, Kılıç AO (2017). Antioxidant phenolic constituents, antimicrobial and cytotoxic properties of Stachys annua L. from both natural resources and micropropagated plantlets. Indian Journal of Traditional Knowledge 16(3):407-416.

Delaquis PJ, Stanich K, Girard B, Mazza G (2002). Antimicrobial activity of individual and mixed fractions of dill, citaro, coriander and eucalyptus essential oils. International Journal of Food Microbiology 74:101-109. https://doi.org/10.1016/s0168-1605(01)00734-6

Dorman HJD, Deans SG (2000). Antimicrobial agents from plants: antibacterial activity of plant volatile oils. Journal of Applied Microbiology 88:308-316. https://doi.org/10.1046/j.1365-2672.2000. 00969.x

Esmaeili AK, Taha RM, Mohajer S, Banisalam B (2015). Antioxidant activity and total phenolic and flavonoid content of various solvent extracts from in vivo and in vitro grown Trifolium pratense L. (Red Clover). BioMed Research International 643285:11. https://doi.org/10.1155/2015/643285

Esmat A, Al-Abbasi FA, Algandaby MM, Moussa AY, Labib RM, Ayoub NA, Abdel-Naim AB (2012). Antiinflammatory activity of Pistacia khinjuk in different experimental models: Isolation and characterization of its flavonoids and galloylated sugars. Journal of Medicinal Food 15(3):278-287. https://doi.org/10.1089/jmf.2011.0099

Falleh H, Jalleli I, Ksouri R, Boulaaba M, Guyot S, Magné C, Abdelly C (2012). Effect of salt treatment on phenolic compounds and antioxidant activity of two Mesembryanthemum edule provenances. Plant Physiology and Biochemistry 52:1-8. https://doi.org/10.1016/j.plaphy.2011.11.001

Giaginis C, Theocharis S (2011). Current evidence on the anticancer potential of Chios mastic gum. Nutrition Cancer 63(8):1174-84. https://doi.org/10.1080/01635581.2011.607546

Golkar P, Taghizadeh M (2018). In vitro evaluation of phenolic and osmolyte compounds, ionic content, and antioxidant activity in safflower (Carthamus tinctorius L.) under salinity stress. Plant Cell Tissue Organ Culture 134:357-368. https://doi.org/10.1007/s11240-018-1427-4

Golkar P, Taghizadeh M, Yousefian Z (2019) The effects of chitosan and salicylic acid on elicitation of secondary metabolites and antioxidant activity of safflower under in vitro salinity stress. Plant Cell, Tissue and Organ Culture 137:575-585. https://doi.org/10.1007/s11240-019-01592-9

Hacibekiroglu I, Yılmaz-Köseoglu P, Nesrin N, Ersin K, Veysel T, Ufuk K (2015). In vitro biological activities and fatty acid profiles of Pistacia terebinthus fruits and Pistacia khinjuk seeds. Natural Product Research 29(5):444-446. https://doi.org/10.1080/14786419.2014.947492

Hatamnia AA, Rostamzad A, Malekzadeh P, Darvishzadeh R, Abbaspour N, Hosseini M, Nourollahi K, Mehr RS. 2016). Antioxidant activity of different parts of Pistacia khinjuk Stocks fruit and its correlation to phenolic composition. Natural Product Research 30(12):1445-1450. https://doi.org/10.1080/14786419.2015.1060593 
Hazrati S, Govahi M, Ebadi MT, Habibzadeh F (2020). Comparison and evaluation of oil content, composition and antioxidant properties of Pistacia atlantica and Pistacia khinjuk grown as wild. International Journal of Horticultural Science and Technology 7(2):165-174. https://doi.org/10.22059/ijhst.2020.287550.316

Hernandez JA, Almansa MS (2002). Short-term effects of salt stress on antioxidant systems and leaf water relations of pea leaves. Physiologia Plantarum 115(2):251-257. https://doi.org/10.1034/j.1399-3054.2002.1150211.x

Holley RA, Patel D (2005). Improvement in shelf-life and safety of perishable foods by plant essential oils and smoke antimicrobials. Food Microbiology 22:273-292. Https://doi.org/10.1016/j.fm.2004.08.006

Hosseinzadeh H, Sajadi Tabassi SA, Milani Moghadam N, Rashedinia M, Mehri S (2012). Antioxidant activity of Pistacia vera fruits, leaves and gum extracts. Iranian Journal of Pharmaceutical Research 11(3):879-887.

Javed R, Gurel E (2019). Salt stress by $\mathrm{NaCl}$ alters the physiology and biochemistry of tissue culture-grown Stevia rebaudiana Bertoni, Turkish Journal of Agriculture Forestry 43:11-20. https://doi.org/10.3906/tar-1711-71

Kaliora AC, Mylona A, Chiou A, Petsios DG, Andrikopoulos NK (2004). Detection and identification of simple phenolics in Pistacia lentiscus resin. Journal of Liquid Chromatography Relation Technology 27:289-300. https://doi.org/10.1081/JLC-120027100

Ksouri R, Megdiche W, Debez A, Falleh M, Grignon C, Abdelly C (2007). Salinity effect on polyphenol content and antioxidant activities in leaves of the halophyte Cakile maritima. Plant Physiology and Biochemistry 45:244-248. https://doi.org/10.1016/j.plaphy.2007.02.001

Mazari K, Bendimerad N, Bekhechi C, Fernandez X (2010). Chemical composition and antimicrobial activity of essential oils isolated from Algerian Juniperus phoenicea L. and Cupressus sempervirens L. Journal of Medicinal Plant Research 4:959-964. http://www.academicjournals.org/JMPR

Mirian M, Behrooeian M, Gahanadian M, Dana N, Sadeghi-Aliabadi H (2014). Cytotoxity and antiangiogenic effects of Rhus coriaria, Pistacia vera and Pistacia khinjuk oleoresin methanol extracts. Research in Pharmaceutical Sciences 10(3):233-240.

Mohagheghzadeh A, Faridi P, Ghasemi Y (2010). Analysis of mount atlas mastic smoke: A potential food preservative. Fitoterapia 81:577-580. https://doi.org/10.1016/j.fitote.2010.01.022

Moreno MIN, Isla MI, Sampietro AR, Vattuone MA (2000). Comparison of the free radical-scavenging activity of propolis from several regions of Argentina. Journal of Ethnopharmacology 71(1-2):109-114. https://doi.org/10.1016/s0378-8741(99)00189-0

Murashige T, Skoog F (1962) A revised medium for rapid growth and bioassays with tobacco tissue cultures. Plant Physiology 15:473-497. https://doi.org.10.1111/j.1399-3054.1962.tb08052.x

National Committee for Clinical and Laboratory Standard (2009). Performance Standards for Antimicrobial Susceptibility Testing; 20th Informational Supplement. CLSI document M100-S19. CLSI, Wayne, PA: NCCSL.

National Committee for Clinical Laboratory Standards (1997). Performance standards for antimicrobial disk susceptibility tests. Approved standard M2-A6. Wayne, PA: NCCLS.

Navarro JM, Flores P, Garrido C, Martinez V (2006). Changes in the contents of antioxidant compounds in pepper fruits at different ripening stages, as affected by salinity. Food Chemistry 96:66-73. https://doi.org/10.1016/j.foodchem.2005.01.057

Ongphimai N (2013). Phenolic acids content and antioxidant capacity of fruit extracts from Thailand. Chiang Mai Journal of Science 40(4):636-642.

Parsaeimehr A, Sargsyan E, Javidnia K (2010). A comparative study of the antibacterial, antifungal and antioxidant activity and total content of phenolic compounds of cell cultures and wild plants of three endemic species of Ephedra. Molecules 15(3):1668-1678. https://doi.org/10.3390/molecules15031668

Rahneshan Z, Nasibi F, Moghadam AA (2018). Effects of salinity stress on some growth, physiological, biochemical parameters and nutrients in two pistachio (Pistacia vera L.) rootstocks, Journal of Plant Interactions 13(1)7:7382. https://doi.org/10.1080/17429145.2018.1424355

Re R, Pellegrini N, Proteggente A, Pannala A, Yang M, Rice EC (1999). Antioxidant activity applying an improved ABTS radical cation decolourization assay. Free Radical Biology and Medicine 26:1231-1237. https://doi.org/10.1016/s0891-5849(98)00315-3

Sacchetti G, Maietti S, Muzzoli M, Scaglianti M, Manfredini S, Radice M, Bruni R (2005). Comparative evaluation of 11 essential oils of different origin as functional antioxidants, antiradicals and antimicrobials in foods. Food Chemistry 91:621-632. https://doi.org/10.1016/j.foodchem.2004.06.031 
Salem MZM, Salem AZM, Camacha LM Ali HM (2013). Antimicrobial activities and phytochemical composition of extracts of Ficus species: An over view. African Journal of Microbiology Research 7(33):4207-4219. https://doi.org/10.5897/AJMR2013.5570

Salem N, Msaada K, Dhifi W, Limam F, Marzouk B (2014). Effect of salinity on plant growth and biological activities of Carthamus tinctorius L. extracts at two flowering stages. Acta Physiologiae Plantarum 36(2):433-445. https://doi.org/10.1007/s11738-013-1424-5

Slinkard K, Singleton VL (1977). Total phenol analyses: Automation and comparison with manual methods. American Journal of Enology and Viticulture 28:49-55.

Taghizadeh SF, Davarynejad G, Asili J, Riahi-Zanjani B, Nemati SH, Karimi G (2018). Chemical composition, antibacterial, antioxidant and cytotoxic evaluation of the essential oil from pistachio (Pistacia khinjuk) hull. Microbial Pathogenesis 124:76-81. https://doi.org/10.1016/j.micpath.2018.08.039

Tahvilian R, Moradi R, Zhale H, Zangeneh MM, Zangeneh A, Yazdani H, Hajialiani M (2016). Ethnomedicinal plants: Study on antifungal activity of essential oil of Pistacia khinjuk (combined with the dominance $\gamma$-Terpinene) against Candida albicans. International Journal of Pharmaceutical and Clinical Research 8(10):1369-1373. www.ijpcr.com

Taran M, Sharifi M, Azizi E, Khanahmadi M (2010). Antimicrobial activity of the leaves of Pistacia khinjuk. Journal of Medicinal Plants 9(6):81-85.

Tilkat E, Ertaş A, Surmuş-Aşan H, Yılmaz MA, Süzerer V (2018). TÜBİTAK KBAG $114 Z 842$ Pistacia lentiscus L.'un 1n vitro sürgün, kallus ve hücre süspansiyon kültürlerinde antikanser aktivite gösteren kimyasal bileşenlerin üretilmesi. [The production of chemical components perfoming anti-anticancer activity in in vitro culture of shoots, callus and suspension of Pistacia lentiscus L.] TÜBİTAK Final Report pp 241 (In Turkish) https://app.trdizin.gov.tr/publication/project/detail/TVRjMk5UTXo=

Tilkat E, Işıkalan Ç, Onay A (2005). In vitro propagation of khinjuk pistachio (Pistacia khinjuk Stocks) through seedling apical shoot tip culture. Propagation of Ornamental Plants 5:124-128. http://www.journalpop.org/2005_5_3_124-128.html

Topçu G, Ay M, Bilici A, Sarıürkcü C, Öztürk M, Ulubelen A (2007). A new flavone from antioxidant extracts of Pistacia terebinthus. Food Chemistry 103(3):816-822. https://doi.org/10.1016/j.foodchem.2006.09.028

Valifard M, Mohsenzadeh S, Kholdebarina B, Rowshanb V (2014). Effects of salt stress on volatile compounds, total phenolic content and antioxidant activities of Salvia mirzayanii. South African Journal of Botany 93:92-97. https://doi.org/10.1016/j.sajb.2014.04.002

Wei T, Sun H, Zhao X, Hou J, Hou A, Zhao Q (2002). Scavenging of reactive oxygen species and prevention of oxidative neuronal cell damage by a novel gallotannin, pistafolia A. Life Sciences 70:1889-1899. https://doi.org/10.1016/S0024-3205(02)01494-7

Yuan G, Wang X, Guo R, Wang Q (2010). Effect of salt stress on phenolic compounds, glucosinolates, myrosinase and antioxidant activity in radish sprouts. Food Chemistry 121:1014-1019. https://doi.org/10.1016/j.foodchem.2010.01.040

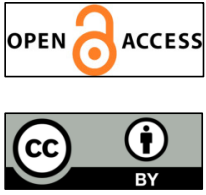

The journal offers free, immediate, and unrestricted access to peer-reviewed research and scholarly work. Users are allowed to read, download, copy, distribute, print, search, or link to the full texts of the articles, or use them for any other lawful purpose, without asking prior permission from the publisher or the author.

License - Articles published in Notulae Botanicae Horti Agrobotanici Cluj-Napoca are Open-Access, distributed under the terms and conditions of the Creative Commons Attribution (CC BY 4.0) License. (c) Articles by the authors; UASVM, Cluj-Napoca, Romania. The journal allows the author(s) to hold the copyright/to retain publishing rights without restriction. 